\title{
Algunas propuestas metodológicas para el estudio de la Baja California durante la segunda mitad del siglo XIX
}

\author{
Aidé Grijalva Larrañaga \\ Centro de Investigaciones Históricas \\ UNAM-UABC
}

\section{Resumen}

La presente constituye una interpretación en términos socioeconómicos de los acontecimientos bajacalifornianos durante la segunda mitad del siglo XIX, vinculando estos sucesos locales a las políticas centrales así como a los intereses extranjeros. Existen dos grandes vertientes de análisis en el estudio de este periodo de la historia bajacaliforniana. La primera vertiente está estrechamente vinculada con el estudio de la historia del sur de California y de los Estados Unidos. La otra vertiente habla de las especificidades que presentaba la península durante el periodo de estudio.

Palabras clave: historia bajacaliforniana, historia sudcaliforniana, línea divisoria internacional.

\begin{abstract}
This is an interpretation on the socioeconomic aspects of the events that took place in Baja California during the second half of XIX century and how were they related to local events, to the federal politics of the capital of the country, and also to foreign interests. To study this frame of time of the history of Baja California there are two main trends for this analysis. We will focus on the first one that is closely linked to researches on the South of California and the United States.
\end{abstract}

Keywords: Baja California history, California history, international boundary. 


\title{
ALGUNAS PROPUESTAS METODOLOGICAS PARA EL ESTUDIO DE LA BAJA CALIFORNIA DURANTE LA SEGUNDA MITAD DEL SIGLO XIX*
}

\author{
Por \\ Aidé Grijalva Larrañaga**
}

Sergio Ortega en su ensayo sobre la periodización de la historia socioeconómica del noroeste mexicano, señala que en las dos últimas décadas del siglo $\mathrm{XIX}$, tienen lugar en dicha región los más acelerados cambios en su historia (Ortega, 1985:14).

El nacimiento de las poblaciones fronterizas del noroeste de México se da, precisamente, a partir de ese momento. Aunque el tratado de Guadalupe Hidalgo definió la nueva frontera desde 1850 , los núcleos o centros de población surgirán la mayoría de las veces, por no decir todas, como producto de los acontecimientos socioeconómicos que tienen lugar, a partir de 1870, en el suroeste de los Estados Unidos (Piñera, 1984:10). La apertura de las últimas fronteras - frontiers - ${ }^{1}$ de los Estados Unidos, la llegada en masa de colonos a la llamada conquista del oeste, el incremento demográfico resultante de ello, tendrán una gran resonancia en la colindante y nueva zona fronteriza y más específicamente en la parte noroeste de México (Rush, 1964).

Se inicia así, lo que Sergio Ortega denomina "el período del noroeste mexicano contemporáneo" (Ortega, 1985:15). En este caso se cumple una de las hipótesis de este autor, en su propuesta para el estudio de la regionalización de la historia mexicana(Ortega, 1980:20). La región fronteriza norte, y sobre todo la noroeste, recibió el fuerte impacto de la inversión norteamericana que enconIró una frontera abierta en su traspatio. Para Jean Revel, la cadena "ferrocarril-compañias mineras-ranchos ganaderos-empresas de irrigación"dio lugar a una transformación del norte de México. Los diversos intereses de los inversionistas norteamericanos indujeron, indudablemente, a diferentes procesos de desarrollo regional. No fue lo mismo una inversión minera que una de tipo agrícola (Revel, 1984:15). Coincidimos con Revel cuando señala que los enclaves fronterizos surgidos en esta época, como los de tipo minero (Cananea), agrícola (valle de Mexicali) y ganadero (Chihuahua), estuvieron ligados a los centros metropolitanos de California, Arizona o Texas (Ibidem). Nosotros agregaremos que la mayoría estaban vinculados a desarrollos similares en sus contrapartes americanas.

\footnotetext{
- Ponencia presentada en el XIII Simposio de Historia y Antropologia de Sonora. Hermosillo, Son., del 17 al 20 de febrero de 1988.

* C'entro de Investigaciones Históricas UNAM-UABCy Seminario de Historiadel Norte de México Ifl Instituto de Investigaciones Históricas UNAM.

' Nos referimos aqui al concepto de "frontera" en el sentido manejado por la escuela historiográfica ueada por Frederick Jackson Turner y sus seguidoresmás distinguidos: Walter Prescott Webb y Ray A Billongton.
} 
Todo esto tiene un papel fundamental para entender tantoel establecimiento en estas nuevas sociedades de una estructura típicamente capitalista, en términos del establecimiento de relaciones sociales de tipo salarial, así como de la tendencia a la integración de las mismas hacia la economía norteamericana más que hacia la mexicana (Piñera, 1982:20 y Ortega, 1985:15).

Por ello, cualquier estudio que abarque la formación y evolución de la zona fronteriza mexicana no debe soslayar la importancia que tiene el conocimiento de la historia de la región fronteriza colindante, así como de los fenómenos socioeconómicos y políticos que influyeron, directa o indirectamente, en la conformación regional de dicha zona.

Angela Moyano ha propuesto una historia comparativa de las relaciones entre Coahuila-Texas, Sonora-Arizona y Baja California-California, como un método para especificar las variaciones regionales existentes en la historia de la frontera norte de México (Moyano, 1984:1 y 2). A manera de ejemplo nos señala que, mientras la relación Sonora-Arizona se vio bloqueada por la mezcla de diversas culturas indigenas que han vivido en sus fronteras, esta situación no se presentó en la relación entre las Californias fronterizas, donde el contacto se dio básicamente entre la población anglosajona y mexicana (fronteriza) (Ibidem).

Esta misma autora ha analizado la interrelación que se ha dado, durante los últimos cien años, entre el sur de California y el norte de Baja California (Moyano, 1983), concluyendo que de dicho análisis le surgió la certeza de "que constituyen una misma región" (Moyano, 1984:2).

David Piñera, el historiador bajacaliforniano, coincide con Angela Moyano. Para entender la historia de la Baja California es indispensable relacionarla con la de California (Piñera, 1982:20). Convencido de que la pujante actividad económica de California se expandió de tal forma sobre Baja California, que en un amplio período del desarrollo histórico de ésta, lo que sucede en ella es un reflejo directo de su vecina, ha propuesto una periodización para el estudio de la historia del norte de la Baja California. En ésta, separa el período comprendido entre 1870 y 1937 , denominándolo "período de integración a la economía californiana". Para Piñera, el desarrollo de la frontera bajacaliforniana se supeditó en forma dependiente al de California de manera tal que terminó por desarticularse del resto del país (Ibidem).

Además, la estrecha vinculación entre ambas Californias no es un fenómeno que se presente a raiz de la delimitación de la nueva frontera. La nueva línea divisoria no sólo no fue suficiente para separar a una región que de antemano vivía procesos históricos imbricad os mutuamente, sino que agudizó la ya tradicional dependencia de la parte norte de la península respecto al sur de California.

La ausencia de comunicación terrestre con el resto del país, la lejanía con La Paz, capital de la península, y el abastecimiento de víveres en el mercado 
californiano sólo propiciaron que, como señala Angela Moyano “de ese momento en adelante, el desarrollo de la Frontera ${ }^{2}$ estuviera supeditado al desarrollo del sur de California" (Moyano, 1984:4).

Sin embargo, aunque coincidimos totalmente con Piñera, cuando señala que la afluencia de inversiones del suroeste de los Estados Unidos al noroeste de México sólo consolidan una serie de antiguas relaciones económicas que la línea divisoria interfirió parcialmente, sin interrumpirlas del todo (Piñera, 1984:20) además de que compartimos el interés de este autor por introducir la variable de la historia californiana como un medio para entender mejor los acontecimientos bajacalifornianos, diferimos de su afirmación de que lo que sucedía en una era reflejo directo de lo que sucedía en la otra.

Consideramos que es necesario ponderar adecuadamente los acontecimientos a ambos lados de la frontera e interrelacionarlos a su vez con los sucesos locales, regionales y nacionales. O sea, lo que Sergio Ortega llama "las condiciones de complementariedad a la sociedad general" de una sociedad regional (Ortega, 1980:17). Esto es, que al mismo tiempo que presenten peculiaridades socioeconómicas y culturales, estas sociedades regionales deben tener también los elementos que las lleven a buscar la complementación con otras sociedades regionales y con su sociedad global. Por esto, no se pueden soslayar los acontecimientos nacionalesque, directa o indirectamente, participan en el surgimiento y posterior evolución de la región que nos ocupa. En el caso de Baja California, es bien sabido que la presencia de capital extranjero fue posible gracias a una política estatal que favoreció abiertamente la penetración de inversión foránea.

Es evidente pues, que dentro de este tipo de estudios regionales, el estudio de la forma de vinculación a la economía norteamericana es fundamental. Para Baja California es necesario además destacar el aislamiento secular de la península, que propició formas y modos de desarrollo económico y social ligados a la supervivencia de la organización misional. Además, es indudable que, a pesar de la débil y frágil vinculación a la economía nacional bosquejada en remedos de organización política, ésta contribuiría finalmente a integrar a la península al Estado-nación mexicano.

Por todo esto, proponemos las siguientes líneas de investigación como una contribución a los incipientes estudios existentes sobre la Baja California durante la segunda mitad del siglo XIX:

Tenemos en primer lugar, la forma en que se da la integración de esta región a la economía norteamericana. Los Estados Unidos viven en esta época los momentos culminantes de expansión de sufrontera como nación. Es el momento de demarcación de sus fronteras políticas. Sin embargo, la expansión de estas fronteras rebasó el trazado de la línea internacional existente a partir de $1850 .^{3}$

\footnotetext{
: Con el nombre de "la Frontera" se cońocia a la parte norte de la peninsula de Baja California aún deshabitada y sin poblaciones conocidas.

' Al respecto se recomiendan los siguientes trabajos: Grebb, 1978: 405-424; Rodman, 1963,
} 
La expansión de la frontera agrícola del suroeste de los Estados Unidos explica que para Baja California el proceso integrador se haya iniciado a través de la apropiación de tierras. Capitalistas norteamericanos descubrieron en su traspatio millones de hectáreas de tierras que podían dar cabida a proyectos de desarrollo agrícola y ganadero y aumentar la frontera agrícola californiana (Rodman, 1973:16-27; Baur, 1951:347-364 y Baur, 1952). Los ranchos californianos se expandian y era necesario buscar nuevas tierras (Gates, 1970:453-472).

Por otra parte, California vive a raíz de la fiebre del oro, un acelerado proceso de desarrollo urbano (Dumke, 1942:425-438; Fritzche, 1972:17-34 y Wright, 1940:323-343), y esto tiene una implicación directa en el norte de la Baja California. El esquema de subdivisión territorial urbano sudcaliforniano fue imitado por los nuevos centros de población fronterizos que surgen del contacto con los núcleos urbanos colindantes. Estos siguen su modelo de desarrollo, estableciéndose lo que Arturo Ranfla denomina "formas integradas de producción entre espacios nacionales compartidos" (Ranfla, 1984:60).

Sin embargo, la expansión de la frontera agrícola sólo fue posible debido a la realización de un conjunto de proyectos hidráulicos que permitieron precisamente dicha expansión. Es lo que en los anales de la historiografía norteamericana se conoce como la reclamation era (Lee, 1978:507-564 y Hundley, 1973:292-325), esto es el momento en que masas de nuevos colonos exigieron -reclamaron - al gobierno norteamericano la ejecución de una serie de obras de irrigación que garantizaran la incorporación de nuevas tierras a desarrollos agropecuarios y urbanos y que sin esas obras seguirían siendo páramos o tierras desérticas. ${ }^{4}$

Estos colonos "reclamaron tierras", pero simultáneamente iniciaron campañas para lograr la ejecución de proyectos hidráulicos de gran magnitud. Estos implicaron inversiones multimillonarias, además de la utilización de las formas más avanzadas de tecnología hidráulica existentes en ese momento y la realización de ambiciosos y arriesgados trabajos de irrigación. ${ }^{5}$

Es un momento de auge en la conquista del oeste. Toda unaépoca: la era de la irrigación (Lee, 1972:289-311). Se llevaron a cabo expediciones, viajes de exploración, investigaciones topográficas. Se reconocieron ramales de ríos, se les recorrió exhaustivamente. ${ }^{6}$ Se emprendieron proyectos aventurados. Se movilizaron influencias políticas; se emprendieron campañas periodísticas, se involucró a la opinión pública y se lograron importantes apoyos financieros guber namentales para la realización de proyectos hidráulicos, que incluían la

\footnotetext{
${ }^{4}$ Como un ejemplo de estopara California se pueden revisar a Rockwood y Heffernan 1930; Hendricks 1971: 1-11; Stowe 1968.

${ }^{5} \mathrm{Al}$ respecto es aleccionante ver la biografía de George Chaffey, escrita por Alexander en 1928.

${ }^{6}$ Esta parte realmente de epopeya está muy bien descrita, sobre todo para el caso de las obras del río Colorado en: Hundley 1966a.
} 
canalización, desviación, derivación o almacenamiento de aguas delos ríos. ${ }^{7}$ Ríos caudalosos, de largos recorridos, fueron domesticados, no sin grandes dificultades. Con esto, miles y miles de hectáreas de tierras fueron abiertas al cultivo.

Es precisamente dentro de este contexto de expansión de frontera agrícola y grandes proyectos hidráulicos, donde surgen las poblaciones del norte de la Baja California. Estas dos historias, la agraria y la hidráulica, vienen a ser las dos grandes coordenadas que establecen y delimitan los espacios en que se desenvuelve el desarrollo inicial de la región que nos interesa (Hundley, 1966b). Es a través de estas dos historias como el proceso de integración a la economía californiana aparece más claramente definido.

La historia del norte de la Baja California es, indudablemente, parte de la historia del sur de California: de la introducción del ferrocarril, de la apertura de nuevas rutas y la aparición de pueblos a lo largo de las vías férreas (Parker, 1937:103-119). De la apertura de las últimas fronteras y de la tenaz lucha de estos nuevos pueblos por asegurarse la dotación de las aguas del río Colorado y con ello, su supervivencia (Schonfeld, 1969:279-425). De la unión del este con el oeste, al construirse el ferrocarril intercontinental en la que varios puntos de la zona se convirtieron en paso obligado del nuevo camino (Lesley, 1939:89-96 y Lesley, 1936).

Nos atrevemos a afirmar que todos los acontecimientos forman parte de una historia común que se entrelaza continuamente. La expulsión de los chinos de California, a raíz de la aprobación de actas inmigratorias, explica la llegada de éstos al norte de la Baja California, donde se convertirían en la mano de obra agrícola. ${ }^{8}$ El desplazamiento del puerto de San Francisco como centro de gravitación comercial y político de California, por el de Los Angeles, ${ }^{9}$ explica el tipo de especulación que se llevó a cabo con los terrenos baldíos de la Baja California, en las últimas décadas del siglo XIX (Hendricks, 1967). El mismo surgimiento del valle de Mexicali sólo se explica como correlato del nacimiento del valle Imperial. Surgen simultáneamente y podemos afirmar que el primero es efecto del segundo. ${ }^{10} \mathrm{~A}$ pesar de esto, ambos valles agrícolas siguieron procesos económicos diferentes que obedecen a sus propias particularidades.

El conocimiento de la historia californiana es, ${ }^{11}$ sin duda, un elemento indispensable para el mejor entendimiento de un conjunto de procesos bajacalifornianos que tienen en ésta uno de sus referentes explicativos.

\footnotetext{
' Los trabajos de William L. Kahrl sobre la desviaciónde las aguas del río Owens para llevar agua a la ciudad de Los Angeles, describen muy bien el ambiente político de la época. Polansky utilizó estas investigaciones como trama central de su película Chinatown. (Véase Kahrl, 1976).

" Sobre esto se pueden revisar los trabajos de Hennings, 1963:291-300; Olin, 1966; Saxton, 1971; Olmsted, 1971: 285-295; y Lorence, 1970.

"Sobre cómo Los Angeles desbancó a San Francisco como principal centro económico del sur de California se puede ver a Gottlieb y Wolt, 1977:11-106; Matson, 1945; Grebb, 1978.

1" Fsto está muy bien descrito en Stowe, 1968; Tout, 1931; también Estrella s/f.

" Para una primera aproximación a este tema es conveniente revisar el artículo de Nash al respecto (Nash, 1981:387-414).
} 
Sin embargo, siguiendo la propuesta metodológica de Sergio Ortega acerca de las condiciones simultáneas de particularidad y complementariedad, las siguientes líneas de investigación se proponen con el fin de destacar estas condiciones para la región bajacaliforniana.

Tenemos así que, para la segunda mitad del siglo XIX, Baja California se enfrentaba a una situación peculiar. Recientemente desgajada de su macizo continental, prácticamente deshabitada, con una nueva frontera donde sus escasos pobladores se aglutinaban en rancherías y alrededor de ex misiones, la región vivía una situación de incertidumbre y desarticulación.

Al impacto de su nueva situación política, se sumaba el de enfrentarse a los esfuerzos realizados por ef gobierno (o los gobiernos) centrales por liderear la integración de las diferentes regiones. Dentro de estos esfuerzos, el proyecto liberal de convertir a México en un país de pequeños propietarios, donde cada uno de sus ciudadanos tuviera acceso a un pedazo de tierra en la Baja California, tuvo efectos que distorsionaron estos objetivos, al menos los declarados.

La ley del 20 de julio de 1863, expedidaen San Luis Potosípor el gobierno de Benito Juárez, y por la cual todo ciudadano mexicano tenia derecho a adquirir y ocupar tierras nacionales hasta por 2,500 hectáreas, fue el instrumento legal ideado por el gobierno liberal para la consecución de su propósito (Dublan y Lozano, 1878, T.9:637-641).

Sin embargo, esta ley tuvo efectos contraproducentes entre los habitantes de la península. Para los viejos pobladores de la Baja California debió ser un mecanismo para legalizar sus posesiones. Pero, por su desconocimiento de la ley y de los tipos de trámites que había que llevar a cabo, se convirtió en fuente de calamidades.

Esta ley incluía una serie de requisitos que para los rancheros y antiguos colonos de la Baja California era casi imposible cumplir. Ejemplos: los artículos 10 y 25 . El primero obligaba a los poseedores de terrenos a mantener un habitante por lo menos cada 200 ha. El artículo 25 preveía que fueracuadrado el terreno adjudicado a los denunciantes (ibidem). Sin embargo, el terreno de la península era, y es aún hoy, inservible para la agricultura. Carece de terrenos aptos para el cultivo, de lluvias permanentes, y de arroyos y manantiales. Muchas veces los terrenos son serranías escabrosas que sólo sirven para la cría del ganado. De esta manera, el propietario que tenía uno o dos sitios de ganado mayor establecía su hogar en el paraje donde encontraba un aguaje y ahí levantaba las chozas de los miembros de su familia, sirvientes y vaqueros. Por todo esto, las medidas de los terrenos se subordinaban a este interés, al así exigirlo la topografía del terreno. 
Había pues, una imposibilidad física e invencible, además de la falta de población, porque entre todos los dueños de los ranchos, ninguno podía sostener el número de individuos exigidos por la ley. ${ }^{12}$

Por ello, a pesar de los esfuerzos que los antiguos residentes de la península llevaron a cabo al respecto, fueron incapaces de asegurar los títulos de las tierras donde habían construido sus ranchos, donde pastaba el ganado - si tenían-y donde habían vivido siempre.

Toda esta situación condujo a los colonos bajacalifornianos a una especie de desamparo legal. Con el advenimiento de las compañías deslindadoras, estuvieron desprotegidos y vulnerables desdeel punto de vista legal, para la comprobación de los derechos sobre sus propiedades. Esto propició que, salvo algunas excepciones, las empresas deslindadoras consideraran que la mayor parte de las tierras de la península eran baldías y por lo tanto susceptibles de ser deslindadas $\mathrm{y}$ adjudicadas en favor de las mismas.

Así pues, aunque la política agraria de los gobiernos liberales estuvo acompañada de serios propósitos de colonización y del convencimiento de que mediante dicho procedimiento se garantizaba el desarrollo económico y social de esa parte del país, los resultados fueron muy distintos. La flexibilidad para conceder tierras en la península dio lugar a un acaparamiento de éstas en unas cuantas manos y el despojo de las mismas a los escasos habitantes de la misma.

Este despojo ha sido poco estudiado. Para el caso de Baja California es prácticamente desconocido. Detectar este tipo de procesos es fundamental para un mejor conocimiento de la historia de esta región. ${ }^{13}$

Otro punto es el relacionado con el despojo de tierras a las comunidades indígenas. Si bien éstas habían desaparecido en la parte sur de la Baja California, se calcula que existían unos 4,000 indígenas viviendo en el norte y que estaban distribuidos por las serranías (rumbos de la sierra de San Pedro Mártir), por los alrededores de Tecate y en las tierras ubicadas en las márgenes de la desembocadura del río Colorado, donde estaba concentrada la mayor parte de la población indígena.

Reportes de la época señalan que, a pesarde que estas comunidades indígenas carecían de formas avanzadas de organización social y económica, estuvieron muy sorprendidos cuando empezaron los trabajos de deslinde por parte de compañías privadas y aun los que iniciaron los ingenieros de la Comisión de Límites después de 1850. El despojo a estas comunidades es un vacío en nuestra historiografía, desconociéndose sus dimensiones y la forma en que se dio.

\footnotetext{
12 "Escrito de los vecinos de Mulegé, firmado por Juan García, A. Villavicencio, Ignacio Villa y Clemente Aguilar al C. Juez Constitucional de Mulegé". Mulegé, B.C. 21 de octubre de 1871, copia mecanografiada localizada en la Colección Pablo Herrera Carrillo de la Universidad de las Américas, Cholula, Puebla.

13 Una primera aproximación a esta problemática se encuentra en Grijalva, 1987.
} 
Por otra parte, existe la idea de que sí hubo resistencia indígena, aunque ésta fue mínima y rápidamente sofocada.

Lo cierto es que a la vuelta del presente siglo dichas comunidades indígenas habían sido diezmadas considerablemente. Una vez más nos enfrentamos a la extinción de comunidades indígenas. Una vez más, grupos étnicos fueron sacrificados en la expansión de nuevas fronteras, probablemente mediante su repliegue, incorporación o su desaparición física.

Otro aspecto a tomar en cuenta está relacionado con la modernización en la medición de las propiedades y el significado de ello. La legislación liberal, al introducir un sistema de medición moderno acabó con el antiguo de herencia española. Las leguas y los sitios de ganado mayor, los solares y las caballerías, dejaron su lugar a las modernas hectáreas, propias del sistema métrico. Las 2,500 hectáreas - equivalentes a 1.42 sitios de ganado mayor-acabaron con esas posesiones que iban de un barranco a otro, de una a otra serranía, de un aguaje al siguiente. Posesiones de formasirregulares, acordes a las posibilidades del terreno, empezaron a ser sustituidas por lotes de forma cuadrada (o lo más parecido a un cuadro) de 2,500 ha cada uno.

Coincidiendo con Witold Kula de que la medida jamás es convencional y que siempre representa un valor, sabemos de que la aparición y propagación del sistema métrico fue vista como el símbolo de la modernización de un país, de la victoria sobre anacronismos feudales (Kula, 1980:14).

El mismo Kula nos señala cómo el sistema métrico fue rodeado de un culto particular: se le consideró racional, perfecto, obra de la mente humana, libre de prejuicios y tradiciones, bueno para todos (Kula, 1980:21). Sin embargo, el sueño de un país equitativamente repartido, cuadriculado ad infinitum chocó con la realidad de un país de geografía incierta. Para el caso de la península de la Baja California, estas pretensiones de la legislación liberal llegaron a ser absurdas: una península semidesértica, con poca agua, prácticamente deshabitada, puso en evidencia el desconocimiento que los gobernantes y legisladores tenían de su país.

Cierto es que detrás de la legislación existía un proyecto de nación; pero desafortunadamente, un proyecto basado en la ignorancia de la multiplicidad regional y de la especificidad local existente a lo largo de esa nación en proyecto.

Para el caso de Baja California, la falta de comunicación, la ignorancia de las disposiciones legales, la poca o nula experiencia de los encargados de ejecutar las leyes, las modificaciones constantes en la legislación agraria, las solicitudes de adecuación de estas leyes a las necesidades concretas de los habitantes de la península, la falta de homogeneidad en las adjudicaciones de tierra, las inconformidades y descontentos derivados de la carencia de criterios y de la ignorancia de la legislación por parte de sus ejecutores, son una prueba de esto que señalamos. 
Todo ello nos demuestra, una vez más, que los esfuerzos legales por sí solos, no logran las transformaciones que pretenden. Es lo que Francois Xavier Guerra llama la relaciónentre la sociedad legal y la sociedad real (Guerra, 1987). Muchas veces, la contrastación de la legislación con la realidad desemboca en la distorsión e incluso en el divorcio de los objetivos y metas anunciados en dicha legislación.

El caso de Baja California es un ejemplo de lo anterior. Pero, si bien es cierto que los propósitos declarados en la legislación liberal (colonización extranjera igual a desarrolto económico) no se lograron, en cambio se dio una de las condiciones básicas del capitalismo: la creación de un mercado interno de tierras. Las tierras bajacalifornianas se transformaron en ambicionada mercancía, en objeto de especulación y de un voraz tráfico. Se incrementóel valor de las mismas y se las incorporó al mercado internacional de especulación de bienes raíces.

Para esto fue necesario llevar a cabo el despojo de sus tierras a los antiguos habitantes, que aunque escasos, obstaculizaban el proceso.

Despojo que nos recuerda al descrito por Marx, en su famoso capítulo de la acumulación originaria. Los "cercados" descritos por él, son sustituidos aquí por las mojoneras que sirvieron para delimitar las propiedades de los nuevos dueños. Las comunidades indígenas y los viejos propietarios de ranchos descubrieron de pronto su incapacidad para demostrar al "reino de la legalidad capitalista", la propiedad de las tierras que habían habitado desde siempre.

Por último, queremos llamar la atención sobre el papel que tuvo el jefe político en todo este proceso. Fueron éstos, los intermediarios entre la población bajacaliforniana y el gobierno del centro. Punto de enlace entre el poder central y el local.

Las peticiones de los pobladores de la península, los informes y comunicados de los jefes y subjefes políticos, fueron motivo de especial atención por parte de los gobiernos centrales. Hubo un empeño de los ministerios correspondientes por satisfacer, en la medida de lo posible, las solicitudes y demandas constantes de los habitantes de la península para lograr el cumplimiento de los requisitos Iegales.

Eran precisamente los jefes políticos los que denunciaban los abusos y desviaciones que se llevaban a cabo en nombre de las nuevas leyes agrarias, los problemas a que se enfrentaban los colonos para regularizar sus posesiones, cnviaban informes de las actividades y de los problemas económicos de la región y, la mayoría de las veces, eran auténticos defensores de los lugareños ante la maquinaria del poder central.

Queremos hacer hincapié en este importante papel que consideramos fundamental para entender el proceso de integración nacional que vivió el país desde los últimos treinta años del siglo XIX. No cabe duda, que, a pesar de los grandes 
obstáculos agudizados por la lejanía, aislamiento e incomunicación con la península, los jefes políticos fueron un vehículo transmisorde opiniones, objetivos, puntos de vista, proyectos y decisiones gubernamentales, así como un catalizador de las inquietudes locales y regionales.

En resumen: consideramos que hay dos grandes vertientes de análisis en el estudio de este período de la historia bajacaliforniana. La primer vertiente está estrechamente vinculada con el estudio de la historia del sur de California y de los Estados Unidos. Las poblaciones del norte de la Baja California aparecen a raíz del trazado de la nueva línea divisoria internacional, y su desarrollo está muy ligado al de las poblaciones sureñas del oeste de los Estados Unidos. Además la situación de frontera aceleróla incorporación de las tierras peninsulares al mercado internacional.

La otra vertiente es la que da cuenta de la especificidad de esa parte de la península: de la escasez de población; del despojo de tierras tanto a los colonos como a las comunidades indígenas radicadas en la región; del papel que juegan las compañías deslindadoras en este despojo; de la resistencia indígena, aunque no se conocen las características de la misma; del importante papel que los jefes y subjefes políticos llevaron a cabo comoelementos de mediación entre el poder central y el local; deldesvirtuamiento de la legislación liberal agraria en cuanto a sus propósitos de colonizar la península con extranjeros.

Sin embargo, éstos son solamente apuntes que se hacen con el propósito de reflexionar sobre este período poco conocido de la historia bajacaliforniana. Invitamos a los que comparten este interés, a discutir algunas de las líneas de investigación aquí propuestas y contribuir a un mejor conocimiento de los procesos históricos regionales del norte de México. 


\section{BIBLIOGRAFIA}

ALEXANDER, J.A. 1928. The Life of George Chaffey, a Story of Irrigation Beginnings in California and Australia, with a foreword by the Rt. Hon. Stanley Melbourne Bruce, introduction by Dr. Elwood Mead and a chapter on the Imperial Valley by H. T. Cory. Mac Millan, Melbourne.

BAUR, John E. 1951. "The Health Seekers and Early Southern California Agriculture", en $T P H R, \mathrm{XX}: 4$. 1952. "Los Angeles County in the Health Rush 1870-1900" en CHSQ; XXXI:1.

DUMKE, Glenn, S. 1942. "The Real State Boom of 1887 in Southern California", en TPHR, XI:4.

DUBLAN y Lozano. 1878. Legislación mexicana o colección completa de las disposiciones legislativas expedidas desde la independencia de la república. Ed. Oficial. Tomo IX. Imprenta del Comercio. México.

ESTRELLA, Gabriel. s.f. "El origen de la región de los valles de Mexicali e Imperial desde la perspectiva de las relaciones sociales". Cuadernos de Ciencias Sociales. Serie 1, № 1. México. Universidad Autónoma de Baja California.

FRITZCHE, Bruno. 1972. "San Francisco 1846-1848: The Coming of The Land Speculator", en $\mathrm{CHQ}, \mathrm{LI}: 1$.

GATES, Paul W. 1970. "The Suscol Principle, Preemption, and California Latifundia", en TPHR, XXXIX:4.

GREBB, Allen G. 1978. "Opening a New Frontier: San Francisco, Los Angeles and the Panama Canal, 1900-1914", en TPHR, XLVII:3.

GRIJALVA, Aidé. 1987. Desventuras y desasosiegos de los colonos bajacalifornianos ante la legislación liberal. 1857-1875. Ponencia presentada en el IV Encuentro de Historia Regional, Culiacán, 3 al 8 de noviembre.

GUERRA, Francoise Xavier. 1987. Conferencia. El Colegio de México. Junio 23.

GOTTLIEB Robert e Irene Wolt. 1977. Thinking Big. The Story of the "Los Angeles Times", its Publishers, and their Influence on Southern California. Putnam's Sons. Nueva York.

HENDRICKS, William Oral. 1971. "Developing San Diego's Desert Empire" The Journal of San Diego History, XVII, 3.

1967. Guillermo Andrade and Land Development on the Mexican. Colorado River Delta, 1874-1905. P.H.D. (History) University of Southern California.

HENNINGS, Robert E. 1963. "James D. Phelan and the Woodrow Wilson Antioriental Statement of May 3, 1912", en CHSQ: XLII, 4.

HUNDLEY, Norris. 1973. "The Politics of Reclamation: California, the Federal Government and the Origins of the Boulder Canyon Act. A Second Look", en $C H Q$ : LII, 4.

1966 (a). Dividing the Waters. A Century of Controversy Between the United States and Mexico. University of California Press: Berkeley y Los Angeles.

1966 (b). The Politics of Water and Geography: California and the Mexican American Treaty of 1944. Ponencia presentada en la reunión de la Pacific Coast Branch American Historical Association, Portland Oregon. Agosto 31. 
KAHRL, William L. 1976. "The Politics of California Water. Owens Valley and the Los Angeles Acueduct, 1900-1927, en $\mathrm{CHQ}$.

KULA, Witold. 1980. Las medidas y los hombres. Ed. Siglo XXI, México.

LEE Lawrence E. 1978. "100 years of Reclamation Historiography”, en TPHR: XLVII, 4 (507-564).

1972. "William Ellsworth Smythe and the Irrigation Movement: a Reconsideration", en TPHR: XLI, 3.

LESLEY, Lewis B. 1936. "A Southern Transcontinental Railroad into California: Texas and Pacific versus Southern Pacific 1865-1885", en TPHR: V. 1939. "The Entrance of the Santa Fe Railroad in California", en TPHR: VIII, 1 (89-96).

LORENCE, James J. 1970. "Business and Reform: the American Asiatic Association and the Exclusion Laws 1905-1907", en TPHR: XXXIX, 4.

MATSON, Clarence Henry. 1945. Building a World Gateway. The Story of Los Angeles Harbor. Pacific Era Publishers. Los Angeles.

MOYANO, Angela. 1983. California y sus relaciones con Baja California (síntesis del desarrollo histórico de California y sus repercusiones sobre Baja California). Sep. 80. F.C.E. México.

1984. Consideraciones acerca del estudio histórico de la Frontera. Ponencia presentada en la Primera Reunión de Historia Regional. Frontera norte. Matamoros, Tamps. Noviembre.

NASH, Gerald D. 1981. "California and its Historians: An Appraisal of the Histories of the State”, en TPHR: L, 4 (387-414).

OLIN Jr. Spencer O. 1966. "European Immigrant and Oriental Alien: Acceptance and Rejection by the California Legislature of 1913", en TPHR.

OLMSTED, Roger. 1971. "The Chinese Must Go!" CHQ: L, 3.

ORTEGA Noriega, Sergio. 1980. "Hacia la regionalización de la historia de México", en Estudios de historia moderna y contemporánea de México.

1985. "Ensayo de periodización sobre la historia socioeconómica del noroeste mexicano, siglos XVI y XIX", en Secuencia: 3.

PIÑERA Ramírez, David. 1982. "Peculiaridades en la periodización histórica de Baja California Norte", en Calafia, IV.

1984. "Tierras deshabitadas y concesionarios extranjeros", en Panorama Histórico de Baja California. UABC.

PARKER, Edna Monch. 1937. "TheSouthern Pacific Railroad and Settlement in Southern California", en TPHR: VI, 2.

RANFLA, Arturo. 1984. "Frontera política y espacio fronterizo", en Estudios fronterizos: I, 4-5.

REVEL, Jean. 1984. "La frontera México-Estados Unidos: mexicanización e internacionalización", en Estudios fronterizos. I, 4-5.

RODMAN, Paul W. 1963. Mining Frontiers of the Far West 1848-1880. New Mexico University.

1973. "The Beginnings of Agriculture in California: Innovation vs. Continuity", in $C H Q$, LII, 1.

ROCKWOOD, Charles Robinson y W.T. Heffernan. 1930. Born of the Desert, by. . . and Personal Recollections of the Early History of Imperial Valley, by Dr. W.T.. . . Calexico Chronicle Pub. 
RUSH, Philip. 1964. A History of the Californians. San Diego.

SHIPEK, F.C. 1965. Lower California Frontier; Articles from the "San Diego Union", 1870. edited by. . . Los Angeles Dawson's Book-Shop. (Baja California Travel Series; 2).

SAXTON, Alexander. 1971. The Indispensable Enemy: Labor and the AntiChinese Movement in California. University of California Press. Berkeley.

SCHONFELD, Robert G. 1969. "The Early Development of California's Imperial Valley", en The Historical Society of Southern California. Part I.

STOWE, Noel. 1968. "Pioneering Land Development in the Californias: An Interview with David Otto Brant", en CHSQ: XLVII.

TOUT, Otis B. 1931. The First Thirsty Years. A History of the Imperial Valley. San Diego.

WRIGHT, Doris Marion. 1940. "The Making of Cosmopolitan California. An Analysis of Immigration, 1848-1870" en CHSQ: XIX, 4. 